\title{
GENOTIPOS G DE ROTAVIRUS DURANTE 10 AÑOS EN NIÑOS CON DIARREA AGUDA DE LA CIUDAD DE LA RIOJA, ARGENTINA. IMPLICANCIAS EN LA VACUNACIÓN
}

\section{DISTRIBUTION OF ROTAVIRUS G GENOTYPES DURING 10 YEARS IN CHILDREN WITH ACUTE DIARRHEA OF THE CITY OF LA RIOJA, ARGENTINA. IMPLICATIONS FOR VACCINATION.}

\author{
María del Carmen Díaz Ariza ${ }^{1}$, Valeria Cuffia ${ }^{1}$, Alejandro Silvera ${ }^{1}$, Natalia Guerra ${ }^{1}$, Cristina Díaz Mayorga ${ }^{1}$, \\ Liliana Recchioni' ${ }^{2}$ Liliana Sabini ${ }^{3}$, Patricia A. Cordoba ${ }^{2,4}$.
}

\section{Resumen:}

La vacunación para rotavirus es obligatoria en Argentina desde enero del 2015. Los genotipos $G$ de rotavirus circulantes previo a la vacunación no fueron estudiados en la Provincia de La Rioja. El objetivo del presente trabajo fue determinar los genotipos $G$ de rotavirus que circulaban en La Rioja en niños con diarrea desde 2000 a 2010. Se determinó la presencia de rotavirus del Grupo A por Inmunocromatografía (Biomerieux) en 418 muestras de materias fecales obtenidas en niños con diarrea aguda, hospitalizados y ambulatorios, en ciudad de La Rioja, Argentina. El $40 \%$ de los niños estudiados provenía del interior de la provincia. Las 90 muestras positivas $(21,53 \%)$ fueron amplificadas por RT-PCR y genotipificadas por Multiplex-PCR para genotipos G1, G2, G3, G4, G9 y G8. Los resultados muestran que el genotipo más frecuente de 2000 a 2003 fue G1 pero que desde 2008 se detectaron los genotipos G2, G3 y G9 en infecciones simples y mixtas. Los genotipos G9 y las infecciones mixta, cuádruple y quíntuple, se presentaron en niños menores de un año de vida.

Este es el primer reporte de los genotipos $G$ de rotavirus en La Rioja. Los resultados indican que los genotipos $G$ circulantes de rotavirus son compatibles con los que se dispone en la vacuna aunque la presencia de infecciones mixtas y genotipo G9 en niños menores de un año sugieren una mayor vulnerabilidad de este grupo para la aparición de cepas con derivas génicas durante la implementación de la vacunación en esta Provincia.

Palabras clave: rotavirus; diarrea; La Rioja; Argentina; Genotipos G.

\section{Abstract:}

Vaccination against rotavirus in Argentina is obligatory from January 2015. From 418 stools in children with acute diarrhea were collected from 2000 to 2010 in city of La Rioja, Argentina, Rotavirus was detected. The 90 rotavirus positive stools samples (21.53\%) were amplified by RT-PCR and genotyped by PCR-Mix for $\mathrm{G1}, \mathrm{G} 2, \mathrm{G} 3, \mathrm{G} 4$, G9 and G8. The results show that during 2000-2003, the most frequent genotype was G1 and but since 2008, the G2, G3 and G9 genotypes in single and mixed infections were detected.

In La Rioja, the vaccines could prevent dehydration in older children the year but children under one year could be vulnerable to the emergence of strains with genic aberrations due to the implementation of vaccination in our region.

Keywords: rotavirus; diarrhea. La Rioja; Argentina; Genotypes G.

\footnotetext{
${ }^{1}$ Instituto Universitario de Ciencias de La Salud. Fundación Barceló H. A. La Rioja

${ }^{2}$ Universidad Nacional de La Rioja

${ }^{3}$ Universidad Nacional de Rio Cuarto

${ }^{4}$ Email de contacto: pcordoba2012@gmail.com
} 


\section{Introducción}

A nivel mundial, rotavirus produce aproximadamente 453.000 de muertes en niños y 2 millones de hospitalizados por deshidratación cada año especialmente menores de 5 años de edad '. En la Argentina rotavirus ocasiona alrededor de 100.000 consultas médicas de niños con la internación del $40 \%$ de los casos ${ }^{2}$. Existen dos vacunas contra rotavirus, licenciadas en Argentina ${ }^{3}$. Una vacuna oral atenuada con cinco virus híbridos bovino-humano de cepa G1, G2, G3, G4, P[8]. La otra vacuna oral atenuada (liofilizada), que contiene una única cepa de origen humano G1P[8]. Esta última se presenta obligatoria a partir de enero del 2015, se suministra por vía oral, en dos dosis, la primera a los 2 meses de vida y la segunda a los 4 meses, y es gratuita y obligatoria ${ }^{4}$.

Rotavirus es un virus desnudo de la Familia Reoviridae constituido por ARN de doble cadena dividido en 11 segmentos. En su cápside externa presenta 2 proteínas, VP7 y VP4, que están relacionadas con la antigenicidad neutralizante del virus, sintetizadas a partir del segmento 9 y del segmento 4 respectivamente. La diversidad de la glicoproteína VP7 genera genotipos G y la diversidad de VP4, genotipos $P 5^{5}$.

En Argentina por estudios previos a la incorporación de la vacuna se determinó que los genotipos frecuentes en la década de los noventa eran G1 seguido de G2 y G3 realizado en las principales ciudades de Argentina, no conteniendo La Rioja ${ }^{2,4,6,7}$. Posteriormente se reportaron la presencia de genotipos G9 y G5 como genotipos de circulación no habituales. Durante el periodo 2009-2011 en Argentina, el laboratorio de vigilancia nacional de rotavirus detectó el $28,5 \%$ de las muestras fecales positivas. Los genotipos $G$ más frecuentes durante esos años fueron G3P[8], G12P[8]; $\mathrm{G} 2 \mathrm{P}[4]^{6}$. Además un genotipo $\mathrm{G} 12$ fue reportado en Buenos Aires ${ }^{8}$.

La implementación de una estrategia de vacunación parece inducir derivas génicas y mecanismos de selección de nuevos virus que podrían interferir en cualquier estrategia de vacunación $n^{9,10,11}$.

En la provincia de La Rioja estudiamos previamente la etiología viral de las diarreas donde el principal agente es rotavirus del Grupo $A^{12}$. Desde el año 2005, la provincia se incorpora a la vigilancia epidemiológica de laboratorio del Ministerio de Salud de la Nación. Previamente analizamos la diferencia de epitopes entre cepas de Rotavirus obtenidos en La Rioja con epitopes de vacunas $\mathrm{G} 1$ y $\mathrm{G} 9^{13}$ pero desconocemos cuales fueron los genotipos de circulación más frecuentes y la característica de los pacientes en la provincia ya que muchos de los estudios de genotipos $G$ de Argentina no fueron realizados en ella. En el presente trabajo estudiamos los genotipo $G$ presentes en 10 años de estudio de rotavirus en la provincia.

\section{Materiales y métodos}

Las materias fecales de 418 niños fueron recolectadas en internados (136 muestras) y ambulatorios (282 muestras) del hospital público regional "Dr. Enrique Vera Barros" y de una clínica privado de la ciudad capital de La Rioja desde el año 2000 hasta el 2010. Los niños tenían entre 1 y 60 meses de edad y el $60 \%$ residían en la capital de la Provincia. En los niños internados la muestra se obtuvo durante las primeras $48 \mathrm{hrs}$ del ingreso y en los ambulatorios se obtuvo en las primeras $72 \mathrm{hrs}$ del comienzo del cuadro clínico. Las muestras positivas por Inmunocromatografía VIKIA® Rota-AdenoBioMeriex fueron procesadas por RT-PCR para amplificar el gen 9 que codifica la proteína VP7 y genotipificadas por Multiplex-PCR para G1, G2, G3, G4, G9 y G8 ${ }^{14}$.

Para el análisis estadístico se realizó las diferencias de promedios de las edades de los niños, según diferentes tipos de genotipos detectados y según los diferentes tipos de infección, se utilizó un modelo de diseño completamente aleatorizado.

\section{Resultados}

La Tabla 1 muestra la detección de rotavirus y genotipos $G$ en las muestras estudiadas. De un total de 418 muestras, 90 fueron positivas correspondiente al $21,53 \%$. A partir del total de muestras positivas, 71 fueron amplificadas (datos no mostrados) y de las cuales 51 muestras pudieron ser genotipificadas correspondiente al $56,7 \%$ de las muestras positivas totales, probablemente por lo poca cantidad de virus en la muestra o porque contenga un genotipo $G$ no analizado en este trabajo. Los genotipos $G$ más frecuentes en los 10 años estudiados fueron $\mathrm{G} 1$, G2, G3, G9 y G4, en orden decreciente de frecuencia. El $37,5 \%$ de las muestras obtenidas en los niños hospitalizados resultaron positivas de las cuales el $72,55 \%$ fueron genotipificadas, a diferencia de las muestras de niños ambulatorios que solo el $35,89 \%$ pudo ser genotipificadas. Los genotipos $G$ en los niños hospitalizados son $G 2$, G3, G1 y G9 en orden decreciente de frecuencia. El genotipo $G$ más frecuentes en los niños 
ambulatorios fue G1, detectándolo junto con G3 y G9. La edad promedio de niños hospitalizados con Genotipos G1 y G9 son menores respecto a los otros genotipos aunque no se detectó significancia con los otros genotipos. En relación a la edad de los niños, se detectó que no existen diferencias significativas según los diferentes genotipos identificados $p=0,1881$.

Tabla 1: Detección de Rotavirus y genotipificación G en muestras de niños con diarrea aguda hospitalizados y ambulatorios desde el año 2000 al 2010 de la ciudad de La Rioja. Edad promedio de niños positivos para rotavirus.

\begin{tabular}{ccccc}
\hline Muestras & $\begin{array}{c}\text { Hospitalizados } \\
\mathrm{n}(\%)\end{array}$ & $\begin{array}{c}\text { Edad } \\
\text { Promedio de niños } \\
\text { hospitalizados }\end{array}$ & $\begin{array}{c}\text { Ambulatorios } \\
\mathrm{n}(\%)\end{array}$ & $\begin{array}{c}\text { Totales } \\
\mathrm{n}(\%)\end{array}$ \\
\hline Recolectadas & $136(100)$ & & $282(100)$ & $418(100)$ \\
Positivas & $51(37.5)$ & $39(13.82)$ & $90(21.53)$ \\
Edad Promedio & $12,55 \pm 6,54$ & & $13,90 \pm 8,01$ & $13,13 \pm 7,20$ \\
X \pm DS & $37(72.55)$ & & $14(35.89)$ & $51(56.67)$ \\
Positivas Genotipificadas & $76^{*}(100)$ & & $12^{*}(100)$ & $88(100)$ \\
Genotipos G detectados & $14(18.92)$ & $8.95 \pm 3.7$ & $10(83.33)$ & $24(27.91)$ \\
G1 & $23(28.38)$ & $14.04 \pm 14.37$ & $0(0)$ & $23(26.14)$ \\
G2 & $18(24.32)$ & $14.5 \pm 16.79$ & $1(8.33)$ & $19(21.59)$ \\
G3 & $13(17.57)$ & $9.62 \pm 5.09$ & $1(8.33)$ & $14(15.90)$ \\
G9 & $8(10.81)$ & $13.62 \pm 15.57$ & $0 / 0)$ & $8(9.09)$ \\
G4 & $0 / 0$ & 0 & $0 / 0)$ & $0)$ \\
G8 & & &
\end{tabular}

* Numero de muestras genotipificadas en niños hospitalizados y ambulatorio en los 10 años de estudio.

No encontramos diferencias significativas en las edades promedio de los niños según los diferentes genotipos identificados p $=0,1881$

La Tabla 2 muestra los genotipos $G$ detectados en cada año de estudio. El genotipo $G$ más frecuente desde 2000 a 2003 fue G1, tanto en hospitalizados como ambulatorios. Durante el 2008 circularon los genotipos G2, G3 y G9 especialmente en hospitalizados. Durante los años 2004 al 2007 hubo pocas muestras positivas y en ninguna caso se pudo genotipificar quizás por falta de virus en las muestras.

Tabla 2: Distribución de genotipos $\mathrm{G}(\mathrm{n}=88)$ de niños con diarrea hospitalizados y ambulatorios por año de estudio en la ciudad de La Rioja desde 2000 al 2010

\begin{tabular}{|c|c|c|c|c|c|c|c|c|c|c|c|c|}
\hline \multirow{2}{*}{ Año } & \multicolumn{6}{|c|}{ Hospitalizados $(n=76)$} & \multicolumn{6}{|c|}{ Ambulatorios $(n=12)$} \\
\hline & G1 & G2 & G3 & G4 & G8 & G9 & G1 & $\mathrm{G} 2$ & G3 & G4 & G8 & G9 \\
\hline 2000 & 3 & 0 & 0 & 0 & 0 & 0 & 0 & 0 & 0 & 0 & 0 & 0 \\
\hline 2001 & 0 & 0 & 0 & 0 & 0 & 0 & 2 & 0 & 0 & 0 & 0 & 0 \\
\hline 2002 & 2 & 1 & 2 & 0 & 0 & 0 & 3 & 0 & 1 & 0 & 0 & 0 \\
\hline 2003 & 1 & 0 & 0 & 0 & 0 & 0 & 5 & 0 & 0 & 0 & 0 & 1 \\
\hline 2004 & 0 & 0 & 0 & 0 & 0 & 0 & 0 & 0 & 0 & 0 & 0 & 0 \\
\hline 2005 & 0 & 0 & 0 & 0 & 0 & 0 & 0 & 0 & 0 & 0 & 0 & 0 \\
\hline 2006 & 0 & 0 & 0 & 0 & 0 & 0 & 0 & 0 & 0 & 0 & 0 & 0 \\
\hline 2007 & 0 & 0 & 0 & 0 & 0 & 0 & 0 & 0 & 0 & 0 & 0 & 0 \\
\hline 2008 & 8 & 19 & 15 & 8 & 0 & 13 & 0 & 0 & 0 & 0 & 0 & 0 \\
\hline 2009 & 0 & 1 & 1 & 0 & 0 & 0 & 0 & 0 & 0 & 0 & 0 & 0 \\
\hline 2010 & 0 & 0 & 0 & 0 & 0 & 0 & 0 & 0 & 0 & 0 & 0 & 0 \\
\hline $\begin{array}{l}\text { Total } \\
\mathrm{n}(\%)\end{array}$ & $14(18.92)$ & $21(28.38)$ & $18(24.32)$ & $8(10.81)$ & $0(0)$ & $13(17.57)$ & $10(83.33)$ & $0(0)$ & $1(8.33)$ & $0(0)$ & $0(0)$ & $1(8.33)$ \\
\hline
\end{tabular}

La Tabla 3 muestra el tipo de infección según la cantidad de genotipos $\mathrm{G}$ encontrados en las muestras de los niños hospitalizados. El genotipo G más frecuente fue G2 con infecciones simples y mixtas donde la edad promedio de los niños fue mayor al año de vida. En niños de la misma edad se determinaron los genotipos G3 y G4 siempre en infecciones mixtas. El genotipo G9 se detectó como el tercero más frecuente con infecciones simples y mixtas con una edad promedio en los niños menor al año de vida. Las infecciones mixtas con 2 o 3 genotipos fueron más frecuentes en niños de edad promedio mayor al año. Las infecciones mixtas con más genotipos (4ó5 genotipos) se presentaron en niños menores al año de vida. Tampoco se detectaron diferencias en edad entre los niños que poseen diferentes tipos de infecciones $\mathrm{p}=0,1491$. 
Tablas 3: Tipos de infección según la cantidad de genotipos G detectados en muestras de niños hospitalizados en La Rioja de 2000 a 2010 en la Provincia de La Rioja.

Tipos de infección

\begin{tabular}{|c|c|c|c|c|c|c|c|c|c|}
\hline \multicolumn{2}{|c|}{ Simple } & \multicolumn{2}{|c|}{ Doble } & \multicolumn{2}{|c|}{ Triple } & \multicolumn{2}{|c|}{ Cuádruple } & \multicolumn{2}{|c|}{ Quíntuple } \\
\hline Genotipos & $\begin{array}{l}\text { Nro. de } \\
\text { muestras }\end{array}$ & Genotipos & $\begin{array}{l}\text { Nro. de } \\
\text { muestras }\end{array}$ & Genotipos & $\begin{array}{l}\text { Nro. de } \\
\text { muestras }\end{array}$ & Genotipos & Nro. de muestras & Genotipos & Nro. de muestras \\
\hline G1 & 10 & G2G3 & 4 & G1G2G3 & 1 & G1G2G3G4 & 1 & G1G2G3G4G9 & 2 \\
\hline G2 & 8 & G2G4 & 1 & G2G3G4 & 1 & G2G3G4G9 & 1 & & \\
\hline \multirow[t]{3}{*}{ G9 } & 1 & G2G9 & 3 & G2G3G9 & 1 & & & & \\
\hline & & G3G9 & 3 & G3G4G9 & 1 & & & & \\
\hline & & G4G9 & 1 & & & & & & \\
\hline Totales (\%) & $19(29.03)$ & & $12(38.70)$ & & $4(12.90)$ & & $2(6.45)$ & & $2(6.45)$ \\
\hline $\begin{array}{c}\text { Edad } \\
\text { promedio }\end{array}$ & $12.33 \pm 6.28$ & & $13.75 \pm 15.03$ & & $19.25 \pm 20.11$ & & $9.50 \pm 4.5$ & & $7.00 \pm 3$ \\
\hline
\end{tabular}

a Los niños con edad promedio menor a los 12 meses de vida corresponden a los que presentan la cepa Genotipo G9 y las infecciones con más de 3 genotipos No se detectaron diferencias de edad promedio entre los niños que poseen diferentes tipos de infecciones $p=0,1491$

\section{Discusión}

En Argentina, se notifican entre 1.100 .000 a 1.250 .000 casos de diarreas agudas por año. El $45-50 \%$ pertenecen a niños menores de 5 años lo que equivale a 490.000 a 550.000 casos de diarreas anuales y a tasas entre 1.450$1.650 / 10.000$ niños menores de 5 años por año. Se estima que la diarrea aguda causa alrededor del $10 \%$ de las internaciones en la primera infancia y entre 60 a 130 muertes anuales $(1,2 \%$ de las muertes en menores de 5 años de edad $)^{3}$. La región NOA, presenta tasas anuales de 3500 casos cada 10.000 niños menores de 5 años, siendo mayor en relación a los valores de media nacional ${ }^{4}$. Los estudios de vigilancia epidemiológica en el país en el análisis por región indican que también en el norte se encuentran los mayores porcentajes de diarrea aguda por rotavirus ${ }^{15,7}$. La Región del NOA estudiada fue Catamarca, Chaco, Corrientes, Formosa, Jujuy, La Rioja, Misiones, Salta, Santiago del Estero y Tucumán con solo 150 muestras positivas durante 2009, 177 en el 2010 y 317 en $2011^{15}$.

En 10 años de estudio nuestros resultados indican que rotavirus es un agente importante de diarreas agudas en la provincia de La Rioja aunque con porcentajes más bajos a los determinados para el noroeste argentino en diferentes años ${ }^{7,15}$. El genotipo G1 fue detectado principalmente en infección simple o mixta con G3 y G9 tanto en pacientes ambulatorios como hospitalizados durante los años 2000 al 2003 confirmando lo observado para otras ciudades de Argentina en estudios de vigilancia epidemiológica nacional ${ }^{2}$. Con posterioridad aunque se procesaron muestras de niños con diarrea ninguna resulto positiva para rotavirus entre los años 2004 a 2007. Durante 2006 se identificaron los genotipos G1, G2, G3, G9 y G8 en la provincia de Córdoba cercana a nuestra región ${ }^{16}$ pero en ningún año de estudio del presente trabajo se detectó genotipo G8 en las muestras. La falta de detección de positivos o de genotipos podría ser resultado de una baja eliminación viral de los infectados o de la sensibilidad de las metodologías utilizadas. En el 2008 se detectaron los genotipos G2, G3 y G9 como más frecuentes. En niños hospitalizados estos genotipos $\mathrm{G}$ se detectaron en más del 50 $\%$ como infecciones mixtas afectando a niños de alrededor del año de vida. Las infecciones mixtas promueven los mecanismos de reordenamiento de genes entre cepas pudiendo producir derivas génicas. En este mismo grupo se determina que el genotipo G9 está presente en niños menores de un año en infecciones simples y mixtas. Este genotipo fue detectado en el país por el laboratorio de vigilancia epidemiológica nacional identificándolo como G9P8 en niños de mayor edad que nuestro estudio ${ }^{17}$. Ese mismo año se observó la presencia de genotipo G12 en la provincia de Buenos Aires ${ }^{8}$. En el presente trabajo no realizamos la genotipificación $\mathrm{P}$ de los rotavirus detectados ni tampoco realizamos la genotipificación para otros genotipos como, G5 y G12 por lo que podría ser parte de las muestras no genotipificadas.

Nuestros resultados indican que los genotipos de las cepas vacunales podrían prevenir la deshidratación en niños mayores del año en la ciudad de La Rioja. Sin embargo cabe destacar que detectamos co-circulación de diferentes genotipos $\mathrm{G}$ y la presencia del genotipo G9, que no forma parte de las cepas vacunales, ambos 
en niños hospitalizados menores del año de vida. Por esta razón consideramos que dicha población es susceptible para la aparición de cepas emergentes como resultado de la presión genética que podría ocasionar la implementación de la vacunación en nuestra región.

\section{Financiamiento}

Este proyecto fue financiado por la Fundación Barceló H.A. Proyecto subsidiado en Convocatoria 2006-2010. Resolución HCS № 3060. Ministerio de Salud de la Provincia de La Rioja durante año 2000-2005

\section{Agradecimientos}

Los autores agradecen a los médicos Giménez Adrián, Hunicken Gustavo, Andrea Toro, Laura Breppre, Carla Peralta Martínez, Pablo Mirabal quienes colaboraron durante algunos momentos de estos 10 años de trabajo. Agracemos la colaboración del Dr. Hector A. Barceló y Dr. Daniel Cordoba.

\section{Bibliografía}

1-Parashar UD, Burton A, Lanata C, Boschi-Pinto $C$, Shibuya K, Steele D, Birmingham M, Glass RI. Global mortality associated with rotavirus disease among children in 2004. J Infec Dis 2009;200(1):S9-S15.

2-Gomez JA, Sordo ME, Gentile A. Epidemiologic patterns of diarrheal disease in Argentina: estimation of rotavirus disease burden. Pediatr Infect Dis J 2002; 21:843-850.

3- Rotavirus. Normas nacionales de vacunación. Ministerio de salud de la Nación. http://www.msal.gob.ar/images/stories/epidemiologia/inmu nizaciones/normas-rotavirus.pdf)

4-Fundamentos de la Introducción de la vacuna contra el Rotavirus. Ministerio de salud 2015.

http://www.msal.gob.ar/images/stories/bes/graficos/000000 0586cnt-2014-12 lineamientos-rotavirus.pdf

5-Estes MK. Rotaviruses and their replication. In: Knipe DM and Howley PM (eds) Fields Virology, 4th edition,. Philadelphia: Lippincott.Raven, 2001, p. 1747-1785

6-Degiuseppe JI, Giovacchini C, Stupka JA. 2009-2011. Vigilancia epidemiológica de rotavirus en la Argentina. Arch Argent Pediatr 2013; 111:148-154

7-Bok K1, Castagnaro N, Borsa A, Nates S, Espul C, Fay O, Fabri A, Grinstein S, Miceli I, Matson DO, Gómez JA. Surveillance for rotavirus in Argentina. J Med Virol 2001;65:190-198.

8- Castello AA1, Nakagomi T, Nakagomi O, Jiang B, Kang JO, Glass RI, Glikman G, Gentsch JR. Characterization of genotype P[9]G12 rotavirus strains from Argentina: high similarity with Japanese and Korean G12 strains. J Med Virol. 2009 Feb;81(2):371-81.

9- Hull JJ, Teel EN, Kerin TK, Freeman MM, Esona MD, Gentsch JR, Cortese MM, Parashar UD, Glass RI, Bowen $M D$, National Rotavirus Strain Suveillance System. United States rotavirus strain surveillance from 2005 to 2008: genotype prevalence before and after vaccine introduction. Pediatr Infect Dis J 2011; 30 (1):S42-47.
10-Kirkwood CD, Boniface K, Barnes GL, Bishop RF. Distribution of rotavirus genotypes after introduction of rotavirus vaccines, Rotarix ${ }^{\circledR}$ and RotaTeq ${ }^{\circledR}$, into the National Immunization Program of Australia. Pediatr Infect Dis J 2011;30 (1):S48-53.

11-Vesikari T, Karvonen A, Ferrante SA, Kuter BJ, Ciarlet M. Sustained efficacy of the pentavalent rotavirus vaccine, $R V 5$, up to 3.1 years following the last dose of vaccine. Pediatr Infect Dis J 2010;29:957-963.

12-Guerra N, Cuffia V, Maldonado X, Díaz Ariza M, Córdoba $P$. Caracterización de diarreas virales en La Rioja. VIII Congreso Argentino de Virología. Resumen Nro.: 10381 Rev Arg de Microbiol. 2005 Vol 37, supl.1 p34

13- Cuffia, V; Díaz Ariza, MdC; Silvera, A, Sabini, L; Cordoba, $P$. Comparison of antigenic dominants of VP7 in G9 and $G 1$ rotavirus strains circulating in La Rioja, Argentina, with the vaccines strains. Viral Immunology. 2016 29(6): 367-371.

14-Gouvea V, $R$ I Glass, $P$ Woods, $K$ Taniguchi, $H \quad F$ Clark, $B$ Forrester, and $Z Y$ Fang. Polymerase chain reaction amplification and typing of rotavirus nucleic acid from stool specimens J Clin Microbiol 1990; 28: 276-282.

15- Degiuseppea J I, Giovacchinib C, Stupkaa J A. Vigilancia epidemiológica de rotavirus en la Argentina: 2009-2011. Arch Argent Pediatr 2013;111(2):148-154 / 148 16-Barril PA1, Giordano $M O$, Isa $M B$, Masachessi G, Ferreyra LJ, Castello AA, Glikmann G, Nates SV. Correlation between rotavirus $A$ genotypes detected in hospitalized children and sewage samples in 2006, Córdoba, Argentina. J Med Virol 2010; 82:1277-1281

17-Mandile MG, Esteban LE, Argüelles MH, Mistchenko A, Glikmann G, Castello AA. Surveillance of group A Rotavirus in Buenos Aires 2008-2011, long lasting circulation of G2P[4] strains possibly linked to massive monovalent vaccination in the region. J Clin Virol 2014 ;60:282-289. 